\title{
The impact of tip bevel angulation on phacoemulsification efficiency and chatter
}

\author{
Isha Gupta, Judd M. Cahoon, Dallas Shi, Cecinio C. Ronquillo Jr, Gareth Gardiner, Jeff H. Pettey, William R. BarlowJr, M. Aabid Farukhi, \\ Brian C Stagg, Brian Zaugg and Randall J. Olson* \\ Department of Ophthalmology and Visual Sciences, John A. Moran Eye Center, University of Utah, 65 Mario Capecchi Drive, Salt Lake City, Utah, 84132, USA
}

\begin{abstract}
Primary objective: To evaluate the effect of tip bevel angulation on phacoemulsification efficiency and chatter.

Research design: In vitro laboratory study.

Methods and procedures: Formalin-soaked porcine lenses were divided into $2 \mathrm{~mm}$ cubes. $0.9 \mathrm{~mm}$ straight $0,15,30,45$ beveled degree tips were used with micropulse ultrasound ( $6 \mathrm{~ms}$ on and $6 \mathrm{~ms}$ off). Power was set at 100\%, vacuum levels were set at $500 \mathrm{mmHg}$; and aspiration rates were set at $50 \mathrm{~mL} / \mathrm{min}$. Efficiency (time to lens removal) and chatter (number of lens fragment repulsions from the tip) were determined.
\end{abstract}

Main outcomes and results: Changing the bevel angulation on a straight $0.9 \mathrm{~mm}$ phacoemulsification tip had no significant effect on efficiency. A 45 degree bevel was the most efficient tip overall. Chatter was seen to be significantly higher with a 15 degree tip (ANOVA, $\mathrm{P}=.0046$ ).

Conclusions: Tip bevel angulation has little effect on phacoemulsification efficiency and chatter, especially when optimized parameters are used. Limitations of this study include use of only one ultrasound power modulation and hard nuclear material.

\section{Introduction}

Phacoemulsification (phaco) is a modern approach to cataract removal and is the preferred method for cataract extraction by eye surgeons. Phaco is highly customizable and computerized, which makes it an attractive option for cataract removal. In addition, the benefits of phaco have long been realized; they include early restoration of sight, safety profile and efficiency. Naturally, current research in this surgical field is focused on just this: enhancing patient outcomes and improving efficiency.

Phaco offers a wide variety of customizable and evolving functions for the surgeon. Bevel tip angle, which refers to the slant of the phaco tip opening, is one of these features. Therefore, bevel tip angulation and its impact on phaco are topics that have been discussed widely. Theoretically, different angles impact surface area available for cataract engagement, ultrasound modality choice, time to occlusion and risk of capsular breakage [1]. DeMill et al. [2] and Oakey et al. [3] have shown that even small adjustments in these parameters can lead to drastic changes in both efficiency and chatter during lens removal; however, evidence for bevel tip angulation and its impact on efficiency and chatter is lacking. Furthermore, when choosing bevel angle, surgeons are often guided principally by personal preference, others by their opinions and training.

Using an in vitro method to study phaco parameters individually, this study looks specifically at the impact of bevel tip angulation on the efficiency and chatter associated with lens removal.

\section{Materials and methods}

S. porcinus lenses were used for this trial, using a model which has been previously described [3]. Pig eyes were purchased directly from a supplier (Visiontech Inc, Sunnyvale, TX, USA) and lenses were dissected away and isolated within 48 hours of arrival to the laboratory. In order to harden lens nuclei, each lens was soaked individually in $10 \mathrm{ml}$ of $10 \%$ neutral buffered formalin for two hours. Following this procedure, lenses were placed in $10 \mathrm{ml}$ of balanced salt solution (BSS) for 24 hours to ensure uniformity and distribution of buffered formalin. Once lenses were prepared, a lens-cutting apparatus was used to cut lenses into uniform $2 \mathrm{~mm}$ cubes. The lens cubes were then placed in a moisture chamber of balanced salt solution. Of note, lenses were considered unusable after 36 hours from cutting. The result of using formalin, and the time interval and methods described above, yield porcine lenses comparable to hard cataractous human lenses (3-4+). Oakey et al. [3] have validated this previously for comparability of both density and behavior to human lenses during phaco.

Prior to the start of trials, all lens cubes were combined and mixed in a single container, which allowed for randomization of cube selection for each trial run. Phaco experimentation was conducted using an Abbott Medical Optics (AMO, Santa Ana, CA, USA) Signature machine. Microsurgical Technology Inc. (MST) (Redmond, Washington, USA) $0.9 \mathrm{~mm}$ straight phaco tips with varying tip bevel

Correspondence to: Randall J. Olson, MD, John A. Moran Eye Center, University of Utah, 65 Mario Capecchi Drive, Salt Lake City, UT, 84132, USA, Tel: 801-5812352; Fax: 801-581-3357; E-mail: randallj.olson@hsc.utah.edu

Key words: chatter, efficiency, micropulse ultrasound, phacoemulsification, tip bevel angulation

Received: January 20, 2017; Accepted: February 08, 2017; Published: February 11,2017 
angles $\left(0^{\circ}, 15^{\circ}, 30^{\circ}, 45^{\circ}\right)$ were used. Other settings used included bottle height set to $50 \mathrm{~cm}$, on time micropulse at $6 \mathrm{~ms}$ and off time at $6 \mathrm{~ms}$, power at $100 \%$, aspiration at $50 \mathrm{ml} / \mathrm{min}$, and vacuum set to $500 \mathrm{mmHg}$. These settings have been shown to produce optimal results in regards to both efficiency and chatter [4-7]. Additionally, because these settings have been optimized, full foot position on pedal (panel mode) was used to standardize results across studies.

Parameters measured included efficiency and chatter. Efficiency was defined as the total time until fragment removal barring any chatter time. Chatter was defined as an event where the lens or remaining fragment was seen to bounce or repulse off the phaco tip. These parameters were measured and recorded in a manner that has been described by Oakey et al. [3].

A random lens cube was removed from the container by one of the authors and placed inside a chamber filled with BSS. The phaco pedal was depressed to engage the lens cube at the phaco tip, and then the pedal was fully depressed to start ultrasound. A stopwatch was used to measure the time from ultrasound start until complete fragment removal. If a cube or fragment was seen to disengage from the tip, time measured was stopped; and again, the pedal was depressed to vacuum until the particle was reengaged. The timer was restarted with initiation of ultrasound. This method allowed for distinction between chatter time delay and time to particle removal. Efficiency was measured in seconds to total lens cube removal by ultrasound.

Efficiency times were averaged and a standard deviation (SD) was calculated. Outliers that were greater than two SDs from the mean were removed from the data set. These outliers likely represent microchatter events, in which the lens fragment fails to fully engage the tip and can be seen to be minimally bouncing on the tip. Microchatter confounds the actual time to particle removal. With these values removed, a new efficiency time mean and SD were calculated. To analyze the final data set, both student's $t$ test and ANOVA analysis were employed.

\section{Results}

Changing the bevel angulation on a straight $0.9 \mathrm{~mm}$ phaco tip had no significant effect on efficiency. There were no significant differences in time to fragment removal between the $0^{\circ}, 15^{\circ}, 30^{\circ}$, and $45^{\circ}$ bevel angled tips. The $45^{\circ}$ bevel was seen to be the most efficient tip overall. However, the difference was minimal and not statistically significant (Figure 1). Chatter was seen to be significantly higher with the $15^{\circ}$ bevel

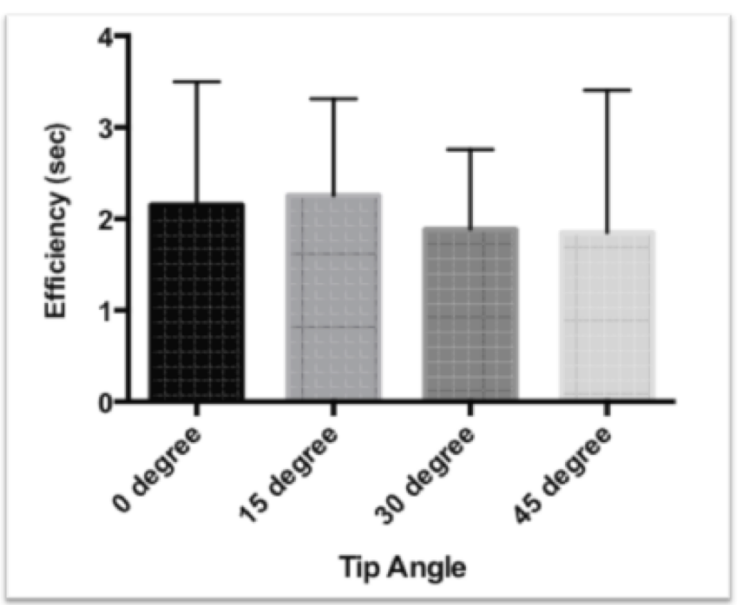

Figure 1. Time required for particle removal reported in seconds $+/$ - standard deviation. angle tip in comparison to $0^{\circ}, 30^{\circ}$, and $45^{\circ}$ angles (ANOVA, $\mathrm{P}=.0046$ ) (Figure 2).

\section{Discussion}

The extent to which bevel tip angulation impacts phacoemulsification efficiency and chatter lacks substantial evidence. Bevel tip angulation has been speculated to influence the time to nuclear removal by influencing the amount of surface area available during cataract engagement. In addition, bevel tip angulation likely influences time to occlusion of tip and risk of capsular breakage, according to Meyer et al. [1]. In a clinical setting and given a wide range of cataract nuclear densities, bevel tip angle impact has been difficult to gauge. Furthermore, surgeons often choose the tip angle based on training, opinions and past experience.

Commonly used bevel tip angles include $0,15,30,45$, and 60 degrees. A 60 degree tip will have a greater slope than one measuring 45 degrees, a 45 degree tip a greater slope than one which measures 30 degrees, etc. The angle of the tip also correlates with surface area as the greater the tip angle, the greater the amount of surface area is available for cataract engagement. Pressure is defined as force per unit area, thereby allowing greater angles to adhere better to nuclear fragments. Conventionally, tips with higher degree angles have been used for removal of denser cataracts. Furthermore, the steeper angles have been considered better for cutting into the nucleus with moves such as grooving, steps which are not considered in this study [8].

Micropulsed ultrasound utilizes longitudinal tip motion with set on and off times. This is important as Olson et al. [9] have suggested that this modality reduces chatter, since the actual on time is brief and therefore minimum momentum is imparted to the lens cube or fragment at the tip. Traditionally, it is thought that the jackhammer mechanism action seen in longitudinal ultrasound modalities optimizes the larger angle tips. The acute angle increases the ability to shave a cataract, thereby making this type of tip most applicable to hard cataracts.

Tip angulation also is thought to play a role in both occlusion and time to lens removal [10]. Smaller angle tips occlude more readily than larger angles. However, surface area increases as tip angle increases, thereby compensating for faster times to occlusion seen with smaller tip angles. Clinically, this may play less of a role as tips occlude when the surface to occlude is parallel to the bevel. The surgeon can and often does manipulate this surface. Probably, what we are seeing is the

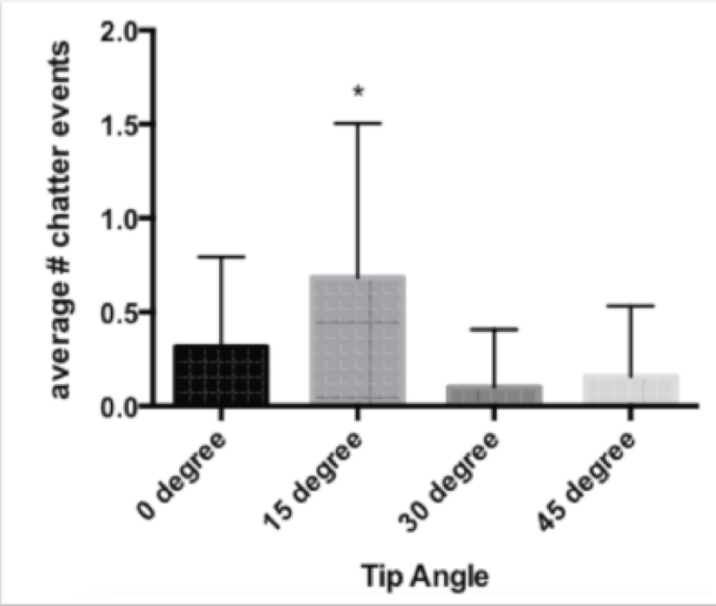

Figure 2. Average number of chatter events during particle removal. * Denotes $\mathrm{P}<.05$ 
counter-balancing forces of ease of occlusion, with the flatter tip angles counter-balanced by greater surface area of occlusion for the steeper angles so that the overall effect is balanced out and the net difference is minimal. Here, with optimized settings and standardized cataract density, this study showed that bevel tip plays an insignificant role in phaco efficiency for lens fragmentation removal.

Inadvertent capsular breakage is an undesirable complication of cataract surgery. While relatively rare, Vasavada et al. [11] have estimated that this complication during posterior polar cataract removal occurs in upward of $26 \%$ of cases. There are multiple variables that influence the likelihood of capsular breakage, needle sharpness and degree of angulation being especially important factors. Further studies will be needed to address the risk of capsular breakage using higher degree of angle bevels; however, it would seem that if the capsule is accidently engaged, a steeper angled tip would create an increased risk for capsular breakage.

A 15 degree bevel tip angle was seen to significantly increase chatter, with all other tips tested showing very little chatter. A flatter angle is readily occluded, while a higher angle increases adherence to cataract materials. 15 degrees is not easily occluded, nor does it have a high adherence rate. We were surprised by these results, although the higher rates of chatter we saw with this tip could possibly be attributed to this phenomenon. A $0^{\circ}$ tip, while not statistically significantly different, did have increased chatter events compared to higher degree angles; this suggests the flatter tips may have a more difficult time digging into the nuclear material so that the full vacuum force can be used to hold the fragment in place.

The in vitro nature of this study is the greatest limitation; however, it is necessary given the practicality and ethics inherent in performing such a study in vivo. It would be nearly impossible to control and evaluate all the variables that would be involved in an in vivo study. We feel that this study has mimicked the clinical situation and optimally isolated the condition being tested.

Other limitations of this study include use of a single ultrasound modality, as well as the size of the lens fragment used. Parameters were optimized for efficiency as per results from previous studies [47], but by no means are these prior studies comprehensive. As more data become available, the methods we used for this study can be easily fine-tuned and then re-evaluated. This study did not assess or address bevel angulation and its effect on impaling and sculpting the nucleus. Anticipated studies include evaluating bevel angulation and its effect on nuclear grooving and risk for capsular breakage.

Bevel tip angulation plays a minimal role in efficiency during phacoemulsification where lens fragments are being removed, which comprises the entire process of chopping techniques. It probably plays a minimum role with chatter, as this was seen only with the 15 degree angle tip. We surmise that hard cataracts, which our model mimics, have a harder time with seating firmly on the phaco tip at this angle, and the result is chatter. This is important new information given the lack of research in regards to bevel tip angulation and its effect on phacoemulsification. Furthermore, this leads the way for customization, with less concern needed when considering tip angulation and especially for those surgeons who use chopping techniques.

\section{Authorship and contributorship}

All authors have made substantial contributions to conception and design, and/or acquisition of data, and/or analysis and interpretation of data; and to drafting the article and/or revising it critically for important intellectual content; and all authors have given her or his final approval of the version to be published. All authors have agreed to act as a guarantor of the work (ensuring that questions related to any part of the work are appropriately investigated and resolved).

\section{Acknowledgment}

Susan Schulman provided assistance with manuscript editing and preparation.

\section{Sources of funding}

This work was supported in part by an unrestricted grant from Research to Prevent Blindness, Inc., New York, New York, USA, to the Department of Ophthalmology and Visual Sciences, University of Utah, Salt Lake City, Utah, USA. Dr. Zaugg is an Achievement Rewards for College Scientists Foundation Scholar (ARCS Foundation Utah, Salt Lake City, Utah, USA). The tips used for this study were donated by Microsurgical Technology, Inc. (Redmond, Washington, USA).

\section{Competing interest}

The authors declare that they have no competing interests.

\section{References}

1. Meyer JJ, Kuo AF, Olson RJ (2010) The risk of capsular breakage from phacoemulsification needle contact with the lens capsule: a laboratory study. $\mathrm{Am} \mathrm{J}$ Ophthalmol 149: 882-886.e881. [Crossref]

2. DeMill DL, Zaugg BE, Pettey JH, Jensen JD, Jardine GJ, et al. (2012) Objective comparison of 4 nonlongitudinal ultrasound modalities regarding efficiency and chatter. J Cataract Refract Surg 38: 1065-1071.

3. Oakey ZB, Jensen JD, Zaugg BE, Radmall BR, Pettey JH, et al. (2013) Porcine lens nuclei as a model for comparison of 3 ultrasound modalities regarding efficiency and chatter. J Cataract Refract Surg 39: 1248-1253. [Crossref]

4. Kirk KR, Ronquillo C Jr, Jensen JD, Zaugg B, Barlow WR Jr, et al. (2014) Optimum on-time duty cycle for micropulse technology. J Cataract Refract Surg 40: 1545-1548. [Crossref]

5. Jensen JD, Kirk KR, Gupta I, Ronquillo C Jr, Farukhi MA, et al. (2015) Determining optimal ultrasound off time with micropulse longitudinal phacoemulsification. $J$ Cataract Refract Surg 41: 433-436. [Crossref]

6. Garff K, Jensen JD, Cahoon J, Gupta I, Stagg B, et al. (2015) Impact of micropulsed ultrasound power settings on the efficiency and chatter associated with lens-fragment removal. J Cataract Refract Surg 41: 1264-1267.

7. Gupta I, Cahoon JM, Gardiner G, Garff K, Henriksen BS, et al. (2015) Effect of increased vacuum and aspiration rates on phacoemulsification efficiency. $J$ Cataract Refract Surg 41: 836-841. [Crossref]

8. Boukhny M (2003) Phacoemulsification tips and sleeves. In: L Buratto, D Apple, L Werner, M Zanini, eds. Phacoemulsification: principles and techniques. 2nd ed. Thorofare, NJ: Slack, Inc. p. 250 .

9. Olson RJ, Kumar R (2003) White Star technology. Curr Opin Ophthalmol 14: 20-23. [Crossref]

10. Fishkind WJ, Neuhann TF, Steinert RF (2010) The phaco machine: the physical principles guiding its operation. In: RF Steinert, ed. Cataract surgery. 3rd ed. Amsterdam: Saunders Elsevier. p.81.

11. Vasavada AR, Mamidipudi PR, Minj M (2004) Relationship of immediate intraocular pressure rise to phaco-tip ergonomics and energy dissipation. J Cataract Refract Surg 30: 137-143. [Crossref]

Copyright: (C2017 Gupta I. This is an open-access article distributed under the terms of the Creative Commons Attribution License, which permits unrestricted use, distribution, and reproduction in any medium, provided the original author and source are credited. 